\title{
The effect of different fatty acid sources on wound healing in rats assessed by matrix-assisted-laser-desorption-ionization mass-spectroscopy-imaging
}

\author{
Tomáš Komprda ${ }^{1}, Z_{\text {Zbyšek Sládek }}^{2}$, Veronika Švehlová ${ }^{1}$, Zuzana Lackováa,4, \\ Roman Gurán̆ ${ }^{3,4}$, Tomáš Do ${ }^{3}$, Jan Wijacki², Marcela Buchtová ${ }^{5}$, Jana Neuwirthová6, \\ Břetislav Gál ${ }^{6}$, Vojtěch Adam ${ }^{3,4}$, Ondřej Zítka ${ }^{3,4}$

\begin{abstract}
${ }^{1}$ Mendel University in Brno, Department of Food Technology, Brno, Czech Republic
${ }^{2}$ Mendel University in Brno, Department of Animal Morphology, Physiology and Genetics, Brno, Czech Republic

${ }^{3}$ Mendel University in Brno, Department of Chemistry and Biochemistry, Brno, Czech Republic

${ }^{4}$ Brno University of Technology, Central European Institute of Technology, Brno, Czech Republic

${ }^{5}$ Czech Academy of Sciences, Institute of Animal Physiology and Genetics, v.v.i., Brno, Czech Republic

${ }^{6}$ Masaryk University, St. Anne's Hospital and Faculty of Medicine, Department of Otorhinolaryngology and Head and Neck Surgery, Brno, Czech Republic
\end{abstract}

Received March 15, 2019

Accepted October 29, 2019

\begin{abstract}
The objective of the present study was to compare the effects of dietary oils containing polyunsaturated fatty acids (PUFA) n-3 and n-6, respectively, on cutaneous wound healing in rats, and to demonstrate the usefulness of the matrix-assisted-laser-desorption-ionization mass spectroscopy-imaging (MALDI MSI) method in this type of experiment. Superiority of PUFA $\mathrm{n}-3$ in this context was the tested hypothesis. Four groups of male Wistar rats by twelve animals each were fed a diet with added 5\% of palm oil (P; control), fish oil (F), Schizochytrium microalga oil (Sch) and safflower oil (S), respectively, for eight weeks. Consequently, dorsal full-thickness cutaneous excisions were performed, and selected markers of wound healing were evaluated 18 days post excision. The median of signal intensity corresponding to an amount of collagen $\alpha-1$ (III) fragment, quantified using MALDI MSI, decreased in a sequence $\mathrm{P}>\mathrm{F}>\mathrm{Sch}>\mathrm{S}$ $(P<0.001)$. Using haematoxylin-eosin staining of the histological preparations, semiquantitatively assessed epithelium height tended to decrease in the order of $\mathrm{P}>\mathrm{S}>\mathrm{Sch}>\mathrm{F}$; the wound extent in the sequence of $\mathrm{P}>\mathrm{Sch}>\mathrm{S}>\mathrm{F}$; and the sequence of the progress of neoangiogenesis was assessed as $\mathrm{S}>\mathrm{P}>\mathrm{F} \approx \mathrm{Sch}$. It was concluded that the tested hypothesis was confirmed only partially: PUFA n-3 showed better results regarding the wound extent, but were inferior in terms of epithelium height and progress of neo-angiogenesis. This was the first time MALDI MSI was successfully employed for evaluating skin wound healing in a rat model.
\end{abstract}

Docosahexaenoic acid, eicosapentaenoic acid, Schizochytrium oil, reepithelialization, MALDI MSI, collagen alpha-1(III)

Wound healing is a dynamic sequential process consisting of three overlapping stages: inflammation, cell proliferation and tissue remodelling (Caetano et al. 2016). The effects of dietary fatty acids, especially long-chain polyunsaturated fatty acids (LC-PUFA n-3) eicosapentaenoic acid (EPA; 20:5 n-3) and docosahexaenoic acid (DHA; 22:6 n-3), known inflammation modulators, on wound healing in animal models are very inconsistent (Komprda 2018): positive effects (Chen et al. 2012; Olson et al. 2013) are contradicted by negative ones (Drzymala-Czyz et al. 2012; dos Santos Rosa et al. 2014), with better results being often obtained with oils containing PUFA n-6 (Otranto et al. 2010).

The main objective of the present study was to evaluate the effect of fish oil (high EPA content) and a Schizochytrium sp. microalga extract (rich in DHA), respectively, in comparison with safflower oil (PUFA n-6 linoleic acid) on selected markers of the healing of a cutaneous excision in rats. A smaller extent of the wound at the given time interval, better reepithelialization and neo-vascularization score by dietary oils containing LCPUFA n-3 was selected as the tested hypothesis. One of the objectives was also to use

Address for correspondence:

Tomáš Komprda

Department of Food Technology

Mendel University in Brno

Zemedelska 1, 61300 Brno, Czech Republic

Phone: +420 545133261

E-mail: komprda@mendelu.cz

http://actavet.vfu.cz/ 
for the first time the cutting-edge method of matrix-assisted-laser-desorption-ionization mass-spectroscopy imaging (MALDI MSI; Angel et al. 2018) for monitoring the spatial distribution of a selected collagen fragment in cutaneous excisions in rats.

\section{Materials and Methods}

\section{Animals and dietary interventions}

Forty-eight adult male rats of the laboratory strain Wistar Albino (Bio Test, Konárovice, Czech Republic) at the age of 8 weeks (the mean live weight of $230 \pm 24.0 \mathrm{~g}$ ) were used. The rats were housed in plastic boxes $(53.5 \times 32.5 \times 30.5 \mathrm{~cm})$ of four animals each in a room maintained at $23 \pm 1{ }^{\circ} \mathrm{C}$, humidity of $60 \%$ and $12 / 12 \mathrm{~h}$ of light/dark cycle (maximum intensity of $200 \mathrm{~lx}$ ). The experiment was performed in compliance with the Czech National Council Act No. 246/1992 Coll. on the protection of animals against cruelty and the amended Act No. 162/1993 Coll., and was approved by the Committee for the Protection of Animals against Cruelty of the Mendel University in Brno (Statement No. 16252-MZE-17214).

The rats were divided into four groups by 12 animals each and fed eight weeks with either the control diet consisting of basic feed mixture (BFM; pelletized complete chow for mice and rats; Biokron, Blučina, Czech Republic) enriched with $5 \%$ of palm oil (P), or one of the three experimental diets: BFM supplemented with 5\% of fish oil (F), $5 \%$ of oil extracted from the Schizochytrium sp. microalga (Sch), and $5 \%$ of safflower oil (S; high content of linoleic acid), respectively. The reason for using the P diet as a control instead of BFM alone was the requirement to keep the diets not only isocaloric, but also isolipidic. Fatty acid composition of the four diets is shown in Table 1.

Table 1. Fatty acid content in the rat diets supplemented with $5 \%$ of palm oil ( $\mathrm{P}-$ control), $5 \%$ of safflower oil (S), $5 \%$ of fish oil (F) and $5 \%$ of Schizochytrium sp. microalga extract (Sch), respectively.

\begin{tabular}{llrrrr}
\hline & \multicolumn{5}{c}{ Diet } \\
\cline { 3 - 6 } & & \multicolumn{1}{c}{$\mathrm{P}$} & \multicolumn{1}{c}{$\mathrm{S}$} & \multicolumn{1}{c}{$\mathrm{F}$} & $\mathrm{Sch}$ \\
\hline Total lipids (\%) & 6.8 & 6.5 & 6.4 & 6.5 \\
& $16: 0$ & 33.0 & 0.1 & 0.5 & 16.2 \\
& $18: 0$ & 4.7 & 3.3 & 5.0 & 3.8 \\
Fatty acid content (\% of the $\Sigma$ & $18: 1 \mathrm{n}-9$ & 36.6 & 17.1 & 39.7 & 22.0 \\
of all determined fatty acids) & $18: 2 \mathrm{n}-6$ & 21.1 & 73.3 & 24.3 & 21.8 \\
& $18: 3 \mathrm{n}-3$ & 0.1 & 0.1 & 0.4 & 0.2 \\
& $20: 5 \mathrm{n}-3$ & 0.2 & 0.5 & 6.3 & 1.0 \\
& $22: 6 \mathrm{n}-3$ & 0.2 & 0.1 & 9.4 & 21.9 \\
PUFA n-6/PUFA n-3 & & 53.9 & 87.6 & 1.7 & 1.1 \\
\hline
\end{tabular}

The diets were prepared (at the laboratory) as follows: pelletized chow was grinded, homogenized with an appropriate amount of the particular oil and cakes weighing approximately $200 \mathrm{~g}$ were prepared by hand. The animals were fed daily ad libitum and had free access to drinking water. Feed consumption was measured daily per given box; daily feed consumption per rat was calculated as a one-fourth of the total intake per cage (due to the ad libitum access to the feed, major differences in consumption between animals in a box were not presumed). The animals were weighed at weekly intervals.

Excisions and sample collection

After 56 days of fattening, all animals were anaesthetized by intramuscular administration of zolazepam (Virbac, Carros, France), and two full-thickness (epidermis, dermis) cutaneous excisions on the dorsum were performed in the sagittal plane using a circular puncher with an internal diameter of $8 \mathrm{~mm}$ (Stiefel Laboratories, Brentford, UK). For technical reasons, the total number of animals was restricted to 48; considering 12 animals the necessary number for sufficient characterization of the dietary group in the healing time period, we gave up the idea of assessing several subsequent healing phases and (based on a preliminary experiment) opted for one 18-day interval. After the excision, the rats were housed individually: two animals were placed in the same plastic box as mentioned above, partitioned with a crossbar into two parts. The wounds were checked daily for infections and since there were none, no additional wound treatment was performed. After 18 days of continuation on the particular diet (P, F, Sch, S), the animals were sacrificed by isoflurane overdosing and the following tissues were taken: two samples of the wounded skin for the production of histological preparations (including MALDI MSI) and a $5 \mathrm{~g}$ aliquot of the liver for determination of fatty acid deposition. 
Fatty acids determination

The content of fatty acids in the diets and in the liver was determined as per our previous study (Komprda et al. 2017).

\section{Histological preparations}

Skin wound samples including ca $2 \mathrm{~mm}$ of skin surrounding the wound were fixed in $10 \%$ buffered formalin, dehydrated by a gradual alcohol series, cleared in xylene, embedded in paraffin blocks and sectioned into the size of 5-8 $\mu \mathrm{m}$ using a rotary microtome Intraco Micro model MT 5505 (Intraco Micro Ltd., Tachlovice, Czech Republic). Sections were subsequently stained with haematoxylin-eosin (H\&E). The histological preparations were then evaluated using a light microscope Olympus BHT S PM1 (Olympus America Inc., USA). Morphometry and evaluation of the qualitative changes were carried out in each sample manually using the ocular micrometer at $\times 5$ (wound extent) and $\times 200$ (reepithelialization extent), magnifications. At least three sections were prepared from each tissue sample. Morphometry was then determined after digitalization of the microscopic preparations by means of the software Lucia (Laboratory Imaging Ltd., Prague, Czech Republic).

\section{MALDI MSI experiment}

Formalin-fixed and paraffin-embedded tissue sections with $8 \mu \mathrm{m}$ thickness, mounted onto indium-tin oxide (ITO) glass slides, were prepared according to Heger et al. (2016).

The mass spectrometry imaging was performed on a MALDI-TOF/TOF mass spectrometer Bruker ultrafleXtreme (Bruker Daltonik GmbH, Bremen, Germany) using a protocol according to Heger et al. (2016). A total sample set spanned 8 ITO glass slides containing 24 tissue sections. As MALDI matrix, $30 \mathrm{mg} / \mathrm{ml}$ 2,5-dihydroxybenzoic acid (DHB) in methanol/water $(50: 50, \mathrm{v} / \mathrm{v})$ with $1 \%$ trifluoroacetic acid (TFA) was used. The raster spot diameter was $30 \mu \mathrm{m}$. MALDI MSI of peptides was performed in a reflector positive mode in the $m / z$ range of $0-5 \mathrm{kDa}$. A total of 500 spectra were summed for each spot using the Random Walk raster pattern, with no evaluation criteria. The $\mathrm{m} / \mathrm{z}$ images were generated and visualized using the SCiLS Lab 2014b software (SCiLS - Bruker Daltonik GmbH, Bremen, Germany). Final MALDI MSI images were prepared in the GIMP 2.8 software.

For on-tissue digestion collagenase A from Clostridium histolyticum was used. Approximately $300 \mu$ of collagenase A solution (1 mg/ml in water) per one slide was applied by ImagePrep (Bruker Daltonik GmbH, Bremen, Germany), the slide was kept inside a small box with humid atmosphere at $37^{\circ} \mathrm{C}$ for $24 \mathrm{~h}$ and the DHB matrix solution was applied. Prior to MSI analysis the slide was dried in a vacuum desiccator for $15 \mathrm{~min}$.

From different types of collagen, collagen III (together with collagen I) are typical for the skin; therefore, collagen alpha-1 (III) chain fragment GAPGFRGPAGPNGIPGEK (according to Oktay et al. 2018) was used for analysis.

\section{Statistical analysis}

The MSI data were evaluated by Anderson-Darling normality test and Kruskal-Wallis test with SCiLS Lab software according to Guran et al. (2017). Histological preparations were only semi-quantitatively assessed, and therefore were not statistically evaluated. Regarding supplementary measurements (weight characteristics, fatty acid deposition in the liver), the differences between dietary groups were evaluated by one-way ANOVA with post hoc Tukey's test using the STATISTICA 12 package (StatSoft, Tulsa, OK, USA).

\section{Results}

\section{Weight characteristics and fatty acid deposition in the liver}

Live weight of the control (P-), S-, F- and Sch-rats after 56 days of fattening (final live weight before excisions) was $536.8 \pm 13.7,507.5 \pm 7.7,519.1 \pm 9.8$ and $521.7 \pm 6.2 \mathrm{~g}$, respectively, and did not differ between dietary groups $(P>0.05)$. Daily weight gains (DWG; within the period until the excision, 56 days) of the P-, S-, F- and Sch-rats are presented in Fig. 1 which shows that, in comparison with control, only safflower oil decreased $(P<0.05)$ DWG of rats. Deposition of EPA+DHA in the liver is shown in Fig. 2. The content of the biologically active substances tested in this main metabolically active organ indicates their availability for the process of wound healing, among other things.

\section{Histological examination}

Semi-quantitatively assessed (H\&E staining) epithelium height decreased in the order of $\mathrm{P}>\mathrm{S}>\mathrm{Sch}>\mathrm{F}$ (Plate III, Fig. 3A). The total extent of the wound decreased in the sequence of $\mathrm{P}>\mathrm{Sch}>\mathrm{S}>\mathrm{F}$ (Plate III, Fig. 3B). Finally, the sequence of the extent of neo-angiogenesis was assessed as $\mathrm{S}>\mathrm{P}>\mathrm{F} \approx \mathrm{Sch}$. Collagen fibres were oriented 


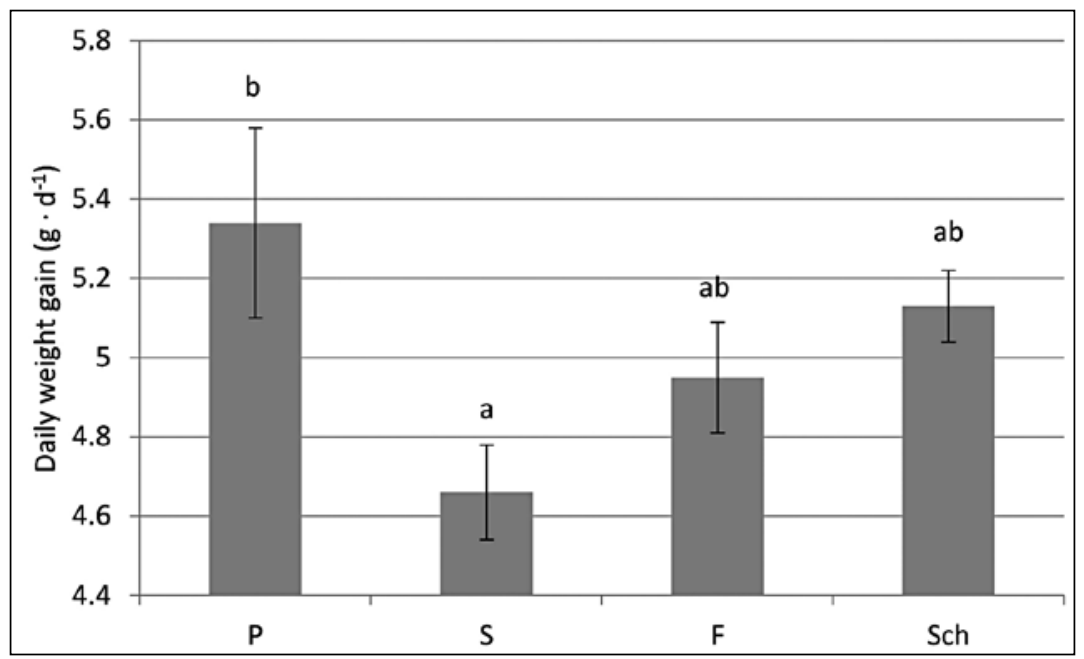

Fig. 1. Daily weight gain of rats fed 56 days a diet supplemented with $5 \%$ of palm oil (P; control), $5 \%$ of safflower oil (S), 5\% of fish oil (F) and 5\% of Schizochytrium sp. microalga extract (Sch), respectively; a, b - means with different letters differ at $P<0.05$, one-way ANOVA with post hoc Tukey's test

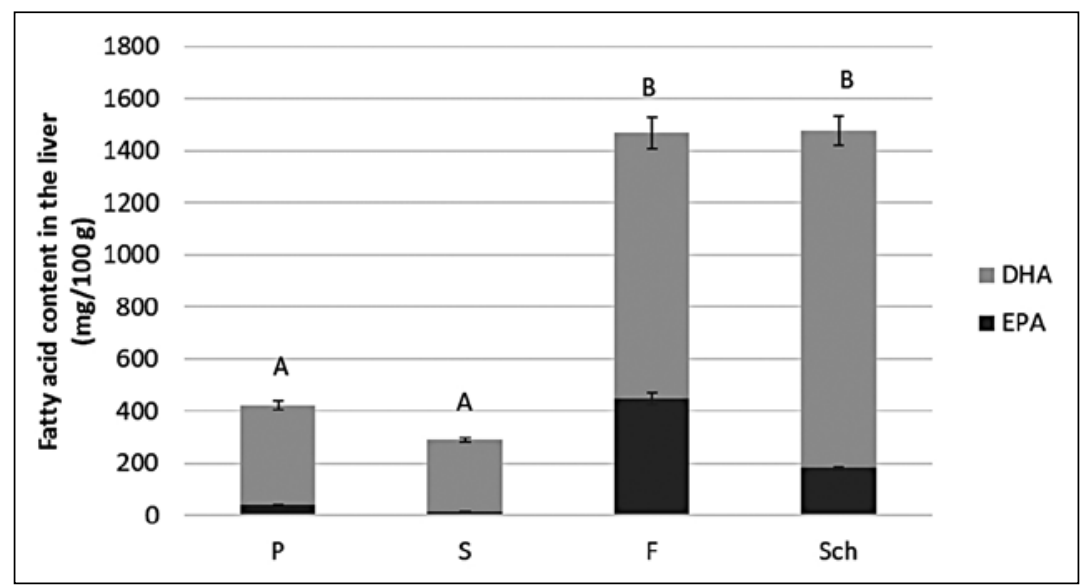

Fig. 2. Deposition of eicosapentaenoic acid (EPA) and docosahexaenoic acid (DHA) in the liver of rats fed a diet supplemented with $5 \%$ of palm oil (P; control), $5 \%$ of safflower oil (S), 5\% of fish oil (F) and $5 \%$ of Schizochytrium microalga extract (Sch), respectively, for 74 days (56 days before +18 days after excision); A, B - means with different letters differ at $P<0.05$ (not individual fatty acids but sums of EPA+DHA are compared); one-way ANOVA with post hoc Tukey's test.

in parallel in the basal part of the granulation tissue of the control samples (Plate III, Fig. 3Ba); the S-preparations (Plate III, Fig. 3Bb) did not differ in this regard. Horizontally arranged collagen fibres switched to an all-direction orientation in the greater depth of the F-preparations (Fig. III 3Bc). The collagen fibres oriented in parallel that were accumulated at the boundary between the granulation tissue and the corium, switched (similar as the F-samples) to an all-direction orientation in the Sch-preparations (Plate III, Fig. 3Bd). 
Dietary fish oil showed more advanced arrangement of collagen fibres and tended to decrease the wound extent 18 days after excision in comparison with control (P) which was a positive finding, but simultaneously tended to worsen the outcome in terms of the epithelium height and a progress of neo-angiogenesis.

MALDI MSI of a collagen fragment

The spatial distribution of collagen alpha-1 (III) chain fragment GAPGFRGPAGPNGIPGEK, m/z $1679.873 \pm 0.100 \mathrm{Da}$, is shown in Fig. 4 (Plate III, Fig. 4). The regions with the highest intensities of this fragment were found in the skin tissue section of control group $P$; the intensities tended to decrease in a row $P>F>S>S c h$. Moreover, the data shown in Fig. 4 (yellow lines) confirmed that the healing process started on the sides of the incision and continued towards the centre. Based on the intensity box plots (Plate IV, Fig. 5), the median of intensity was decreasing $(P<0.001)$ in a sequence $\mathrm{P}>\mathrm{F}>\mathrm{Sch}>\mathrm{S}$. Therefore, the output of the MALDI MSI measurements differed somewhat from the histology data.

\section{Discussion}

The effect of dietary oil on the weight characteristics of rats

The reported effects of PUFA n-3 on DWG of rats are contradictory: no effect of dietary fish oil (Campioli et al. 2012); a tendency to increase DWG in the F-rats in comparison with safflower oil (this study; Fig. 1); a significant DWG decrease in the Sch-fed rats in comparison with safflower oil (Komprda et al. 2016). The contradiction corroborates the conflicting epidemiological evidence of a presumed inverse association between consumption of LC-PUFA n-3 and obesity (Howe et al. 2014).

\section{Histological evaluation}

Gercek et al. (2007) reported significantly smaller mean wound depth but lower mean scores for epidermal and dermal regeneration, granulation tissue thickness and angiogenesis in rats treated with parenteral fish oil emulsion 18 days after the skin wounding in comparison with the control, which was very similar to our results.

On the other hand, the percentage of wound closure seven days after the excision on the back of rats was higher in control than in the fish oil-fed group in an experiment by Otranto et al. (2010). The percentage of re-epithelialized wound area 18 days post excision was not different between the sunflower oil and fish oil group, but was lower in the fish oil group than in the control. These data partially correspond with the results of the present experiment, where the height of the epithelium in the F-rats tended to be lower in comparison with the control group.

Otranto et al. (2010) also reported delayed angiogenesis and impaired formation of the granulation tissue in the fish oil group and in the sunflower oil-fed rats; results of the present experiment are partially in accordance with the quoted study: we can also infer a better score of dietary safflower oil than fish oil as far as re-epithelialization and vascularization is concerned.

\section{Mapping of collagen distribution by MALDI MSI}

The most recent and actually the only study in which MALDI MSI was used in wound healing evaluated the distribution of lipids involved in the skin barrier repair/wound healing process using an in vitro living skin equivalent model (Lew is et al. 2018). However, the animal (rat) model used in the present study is more suitable than in vitro models regarding the relevance of gained information to natural biochemical processes occurring in a living organism. 
The use of formalin-fixed and paraffin-embedded (FFPE) tissue sections for MALDI MSI has its limitations regarding the processes of deparaffinization and antigen retrieval, during which the analytes of interest can be diluted and more delocalized in the tissue. Also, the efficiency of their ionization is usually worse than in the case of cryosections (Pietrowska et al. 2016).

It can be concluded that the hypothesis tested in the present study was only partially confirmed: the results show ambiguous effects of oils containing PUFA n-3 on the histological markers, including their inconclusive effect on the weight characteristics of rats. On the other hand, the present experiment confirmed the power of the MALDI-TOF MSI technique, which was applied for the first time in the rat skin healing experiment. In order to increase effectivity of PUFA n-3 in wound healing, the usefulness of their topical application in a combination with nanoparticles would be worthy of testing in future experiments.

\section{Acknowledgements}

The experiment was financially supported by the Internal Grant Agency of Mendel University in Brno (project no. TP3/2017) and by CEITEC 2020 (LQ1601).

\section{References}

Angel PM, Comte-Walters S, Ball LE, Talbot K, Mehta A, Brockbank KGM, Drake RR 2018: Mapping extracellular matrix proteins in formalin-fixed, paraffin-embedded tissues by MALDI imaging mass spectrometry. J Proteome Res 17: 635-646

Caetano GF, Fronza M, Leite MN, Gomes A, Frade MAC 2016: Comparison of collagen content in skin wounds evaluated by biochemical assay and by computer-aided histomorphometric analysis. Pharm Biol 54: 2555-2559

Campioli E, Rustichelli C, Avallone R 2012: N-3 dietary supplementation and lipid metabolism: Differences between vegetable- and fish-derived oils. J Funct Foods 41: 207-212

Chen WY, Lin SY, Pan HC, Liao SL, Chuang YH, Yen YJ, Chen CJ 2012: Beneficial effect of docosahexaenoic acid on cholestatic liver injury in rats. J Nutr Biochem 23: 252-264

dos Santos Rosa A, Bandeira LG, Monte-Alto-Costa A, Romana-Souza B 2014: Supplementation with olive oil, but not fish oil, improves cutaneous wound healing in stressed mice. Wound Repair Regen 22: 537-547

Drzymala-Czyz S, Banasiewicz T, Tubacka M, Tarasiuk-Rusek A, Majewski P, Drews M. Walkowiak J 2012: Discrepancy between clinical and histological effects of DHA supplementation in a rat model of pouchitis. Folia Histochem Cyto 50: 125-129

Gercek A, Yildirim O, Konya D, Bozkurt S, Ozgen S, Kilic T, Sav A, Pamir N 2007: Effects of parenteral fish-oil emulsion (Omegaven) on cutaneous wound healing in rats treated with dexamethasone. J Parenter Enter Nutr 31: $161-166$

Guran R, Vanickova L, Horak V, Krizkova S, Michalek P, Heger Z, Zitka O, Adam V 2017: MALDI MSI of MeLiM melanoma: Searching for differences in protein profiles. PLOS ONE 12: e0189305

Heger Z, Polanska H, Merlos Rodrigo MA, Guran R, Kulich P, Kopel P, Masarik M, Eckschlager T, Stiborova M, Kizek R, Adam V 2016: Prostate tumor attenuation in the nu/nu murine model due to anti-sarcosine antibodies in folate-targeted liposomes. Sci Rep 6: 33379

Howe PRC, Buckley JD, Murphy KJ, Pettman T, Milte C, Coates AM 2014: Relationship between erythrocyte omega-3 content and obesity is gender dependent. Nutrients 6: 1850-1860

Komprda T 2018: Effect of n-3 polyunsaturated fatty acids on wound healing using animal models - a review. Acta Vet Brno 87: 309-320

Komprda T, Rozikova V, Zamazalova N, Skultety O, Vicenova M, Trckova M, Faldyna M 2017: Effect of dietary fish oil on fatty acid deposition and expression of cholesterol homeostasis controlling genes in the liver and plasma lipid profile: comparison of two animal models. J Anim Physiol Anim Nutr 101: 1093-1102

Komprda T, Sladek Z, Skultety O, Krizkova S, Rozikova V, Nemcova B, Sustrova T, Valova M 2016: Effect of dietary Schizochytrium microalga oil on selected markers of low-grade inflammation in rats. J Anim Physiol Anim Nutr 100: 1169-1178

Lewis EEL, Barrett MRT, Freeman-Parry L, Bojar RA, Clench MR 2018: Examination of the skin barrier repair/ wound healing process using a living skin equivalent model and matrix-assisted laser desorption-ionizationmass spectrometry imaging. Int J Cosmetic Sci 40: 148-156

Oktay HO, Cenker E, Zeynep A, Filiz O, Mutay A 2018: Mass spectrometric identification of collagen alpha-1 (III) chain and chondroitin sulfate proteoglycan-4 nitration in patients with acute pulmonary embolism. Curr Proteomics 15: 34-41

Olson MV, Liu YC, Dangi B, Zimmer JP, Salem N, Nauroth JM 2013: Docosahexaenoic acid reduces inflammation and joint destruction in mice with collagen-induced arthritis. Inflamm Res 62: 1003-1013 
Otranto M, Do Nascimento AP, Monte-Alto-Costa A 2010: Effects of supplementation with different edible oils on cutaneous wound healing. Wound Repair Regen 18: 629-636

Pietrowska M, Gawin M, Polanska J, Widlak P 2016: Tissue fixed with formalin and processed without paraffin embedding is suitable for imaging of both peptides and lipids by MALDI-IMS. Proteomics 16: 1670-1677 
Plate III

Komprda T. et al.: The effect of different ... pp. 443-449

A

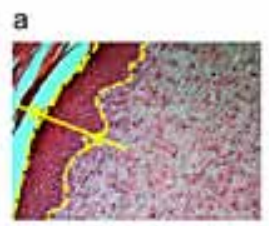

c

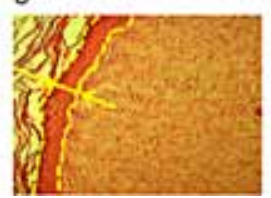

b

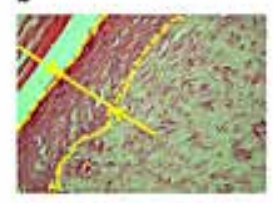

d

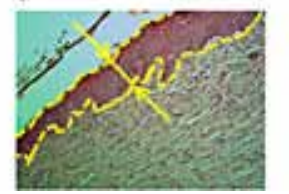

B

a

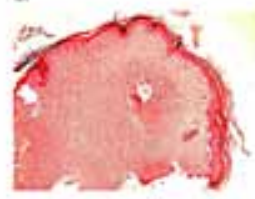

c

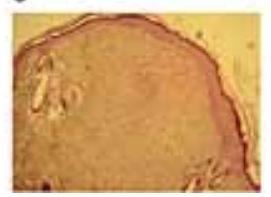

b

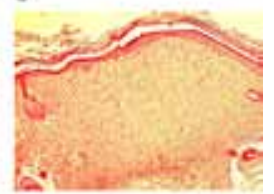

d

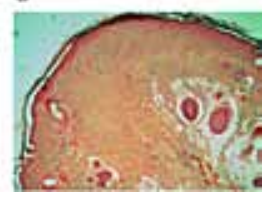

Fig. 3. Reepithelialization (A) and wound extend (B) 18 days post excision in rats fed a diet supplemented with $5 \%$ of palm oil (P; a), 5\% of safflower oil (S; b), 5\% of fish oil (F; c) and 5\% of Schizochytrium microalga extract (Sch; d), respectively for 74 days ( 56 days before +18 days after excision). Haematoxylin\& eosin staining. Magnification was $\times 200$ for $(A)$ and $\times 5$ for (B). The epithelium and its width is highlighted by yellow dashed lines and arrows

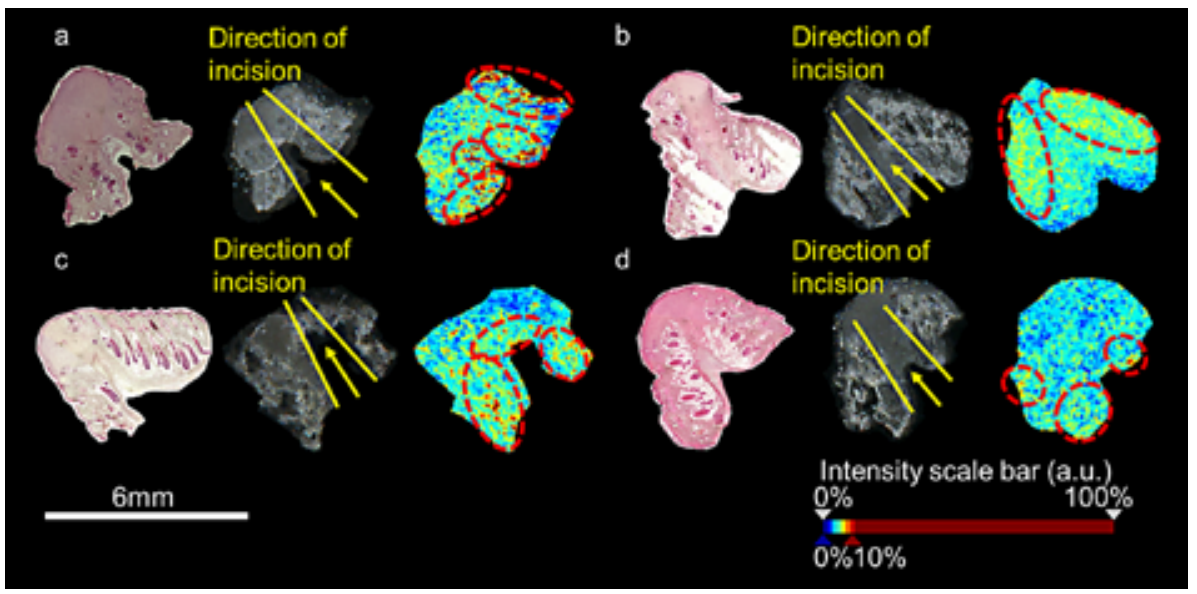

Fig. 4. Haematoxylin-eosin images combined with matrix-assisted-laser-desorption-ionization-time-offlight mass spectroscopy imaging of the $\mathrm{m} / \mathrm{z} 1679.873 \pm 0.100$ Da collagen alpha-1(III) chain fragment GAPGFRGPAGPNGIPGEK, in the dermis 18 days post-excision in rats fed ( 56 days before +18 days after excision) a diet supplemented with $5 \%$ of palm oil (P - control; a), 5\% of safflower oil (S; b), 5\% of fish oil (F; c) and 5\% of Schizochytrium microalga extract (Sch; d), respectively. The regions with the highest intensity of $\mathrm{m} / \mathrm{z} 1679.873 \pm 0.100 \mathrm{Da}$ fragment are highlighted by red ovals 


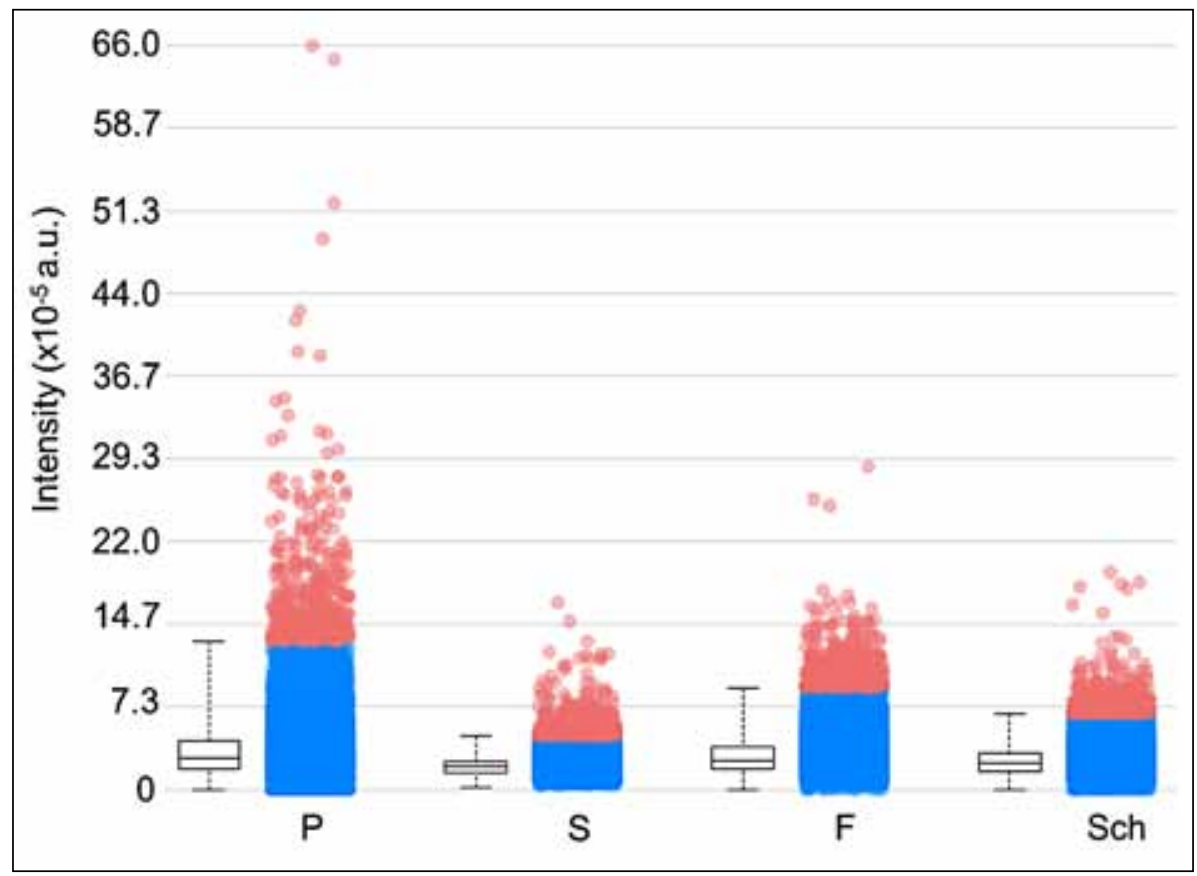

Fig. 5. Intensity box plot for the $\mathrm{m} / \mathrm{z} 1679.873 \pm 0.100$ Da collagen $\alpha-1$ (III) chain fragment GAPGFRGPAGPNGIPGEK in the tissue sections of the dermis 18 days post excision in rats fed (56 days before +18 days after excision) a diet supplemented with $5 \%$ of palm oil ( $\mathrm{P}-$ control $), 5 \%$ of safflower oil (S), 5\% of fish oil (F) and 5\% of Schizochytrium sp. microalga extract (Sch), respectively; each dietary group is represented by six tissue sections, several tens of thousands of the mass spectra were measured within each section, and intensity of each mass spectrum is depicted by a spot within the plot; the intensity decreased in the order of $\mathrm{P}>\mathrm{F}>\mathrm{Sch}>\mathrm{S}(P<0.001$; Kruskal-Wallis test $)$ 